\title{
Research on load transfer based on power supply unit of distribution network
}

\author{
Tianchen $\mathrm{Wu}^{1, *}$, Jun $\mathrm{Han}^{2}$, Chao Cai ${ }^{2}$, Anjie $\mathrm{Fan}^{2}$ \\ ${ }^{1}$ School of Electrical Engineering, Southeast University, China \\ ${ }^{2}$ State Grid Jiangsu Economic Research Institute, China
}

\begin{abstract}
In this paper, the medium voltage distribution network is used as the research object, and the NSGA-II algorithm is used to realize efficient search of load transfer routes. The distribution network topology is formed by the CIM information model, and the load groups carried by each section of the line are calculated. By simulating a fault, determine the power outage area, search for the load transfer path of the adjacent non-faulted power loss area, and combine different load sections to perform power flow calculation, verify whether the equipment load on the transfer channel exceeds the limit, and determine whether the transfer path is effective. The entire optimization process utilizes the fast non-dominated sorting and crowding comparison operators of the NSGA-II algorithm. While maintaining the diversity of the optimal population, through the elite strategy, the sampling space is expanded, so that the optimal process is more evenly distributed in the solution space.
\end{abstract}

\section{Introduction}

In recent years, the problem of load transfer for research scholars at home and abroad a large number of studies, a series of research results: in terms of the research object, mainly "bus/branch" model, for a single radiant power supply structure, and the actual distribution circuit to the node/switch model, for more than a single power supply structure radiant network contact each other, so the practicability of the current study was poor $[1,2]$.

In the method of solving the load transfer problem, heuristic algorithms and intelligent optimization algorithms are mainly used [1-4]. The load transfer problem belongs to the research category of distribution network reconfiguration optimization, and it is a highly complex mixed integer, nonlinear, multi-objective programming problem. The heuristic algorithm generally sets certain rules to limit the transfer conditions, thereby reducing the search solution space and quickly searching for the transfer path. This method is convenient and easy to understand, and the calculation speed is fast, but it lacks the ability to find the global optimal solution. For lines with a higher load rate, the maximum recovery of the outage load cannot be guaranteed or a poor transfer plan can be given.

Artificial intelligence algorithms have the characteristics of faster optimization speed and better convergence. Among them, genetic algorithm simulates the evolutionary theory of biology and is a parallel and random adaptive optimization algorithm. Through the design of chromosome locus, through duplication, crossover and mutation operations, the excellent genes "most suitable for the environment" are calculated, which has good global convergence. Coding is the core of genetic algorithm and directly affects the scale of the solution. Traditional binary coding will cause the scale of the solution to grow exponentially. The optimized real number coding method greatly reduces the solution space, but there are still a large number of infeasible solutions. For research models, traditional genetic algorithms often convert multi-objective models into single-objective models for solution, and cannot accurately compare the pros and cons of various indicators.

This paper proposes a real number coding method based on the distribution network loop adapted to the wiring unit. Under the circumstance of guaranteeing the maximum load recovery, the optimization target is based on the number of switching actions, network loss, and voltage deviation. Using NSGA-II as the optimization method, the optimal transfer path is calculated. While maintaining the diversity of the optimization population, through the elite strategy, the sampling space is expanded, so that the optimization process is more evenly distributed in the solution space.

\section{Mathematical model of load transfer}

\subsection{Model transformation}

The topology structure of the actual distribution network connection group belongs to the node/switch model, which is reflected by the CIM information model, mainly including the information of the distribution network equipment and its connection relationship. By

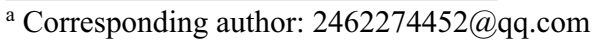


matching the terminal number of the equipment, the connection of bus, switch, knife brake, circuit and other equipment is formed. This model is the basis of routing path search.

To determine whether the switching path is valid, the node/switch model needs to be transformed into the bus/branch model. By combining node/switch model and switch state information, terminal Numbers on both sides of non-impedance equipment (closed switch, knife switch, etc.) are merged. The model is the basis of power flow calculation.

\subsection{The objective function}

The selected objective function of load transfer mainly includes network loss, voltage deviation rate and switching times.

\subsubsection{Network loss}

Taking the minimum loss of power distribution network as the objective function can effectively improve the economy of system operation. The typical expression of the objective function is:

$$
\min f=\sum_{i=1}^{n} x_{i} r_{i} \frac{P_{i}^{2}+Q_{i}^{2}}{U_{i}^{2}}
$$

Where, $x_{i}$ is the opening and closing state of the switch, 0 means opening and 1 means closing. $r_{i}$ is the resistance of the branch $i, P_{i}$ and $Q_{i}$ are the active power and reactive power flowing through the branch $i$. $U_{i}$ is the voltage at the end nodes of branch $i$, and $n$ is the total number of branches.

\subsubsection{Voltage deviation rate}

Taking the minimum voltage deviation rate of each node as the objective function can improve the reliability of power supply and guarantee the power quality. The typical expression of the objective function is:

$$
\min f=\sum_{j=1}^{m}\left|U_{j}^{\prime}-U_{N}\right|
$$

Where, $U_{N}$ is the standard voltage, $U_{j}^{\prime}$ is the voltage of nodes after load transfer, and $m$ is the total number of nodes.

\subsubsection{Switch operation times}

The more switching times of segment switches and contact switches in distribution network, the greater switch loss will affect the service life. Frequent operation switch is unfavourable to fault recovery and increases the time of fault recovery. The typical expression of the objective function is:

$$
\min f=\sum_{i=1}^{N 1}\left(1-x_{i}\right)+\sum_{j=1}^{N 2} y_{j}
$$

Where, ${ }^{x_{i}}$ and $y_{j}$ are the opening and closing states of segment switch and contact switch respectively, 0 when disconnected and 1 when closed. $N 1$ and $N 2$ are respectively the total number of segment switches and the total number of contact switches.

\subsection{Constraint condition}

\subsubsection{Power flow constraint of distribution network}

In the process of distribution network operation, voltage, current and power should meet the constraints of power flow.

\subsubsection{Distribution network topology constraints}

The distribution network should ensure that its structure is radiant.

\subsubsection{Voltage and current constraints}

There are upper and lower limits for the nodal voltage of the line. If the limits are exceeded, the system will become unstable. Its constraints are as follows:

$$
U_{i \min }<\left|U_{i}\right|<U_{i \max }
$$

Where, $U_{i \min }$ is the lower limit value of node voltage, and $U_{i \max }$ is the upper limit value of node voltage.

The line has the maximum allowable current, and the current shall not exceed the limit value:

$$
I_{n} \leq I_{n \max }
$$

Where, $I_{n}$ is the line current. $I_{n \max }$ is the maximum allowable current of the line.

\section{Mathematical model of load transfer}

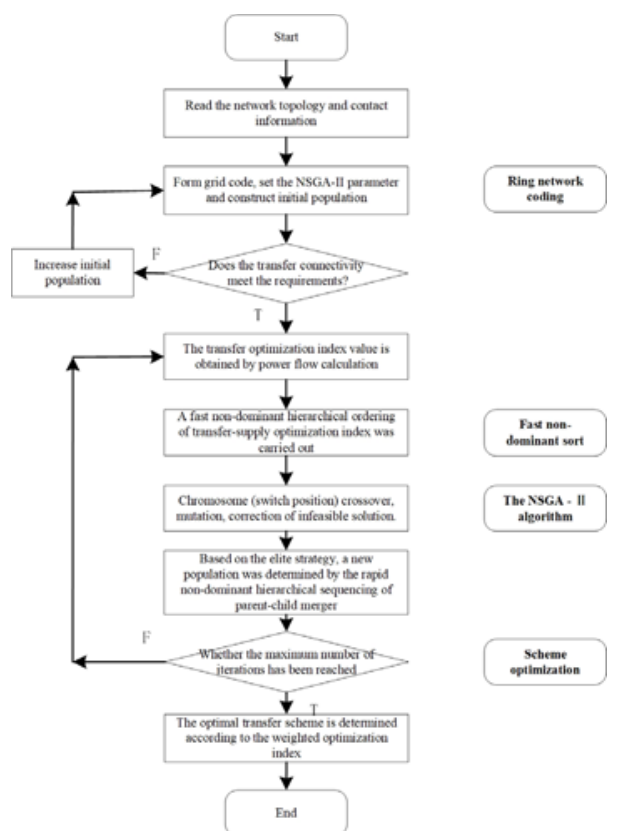

Figure 1. Flow chart of load transfer based on NSGA-II algorithm. 
The load transfer flow chart based on the NSGA-II algorithm is shown in Figure 1.

\subsection{Identify the loss of power area}

It is the basis of load transfer analysis to determine the electrification/loss range of distribution network caused by various faults. The distribution network is divided into three types: live area, fault/maintenance loss area, and non-fault/maintenance loss area. According to the distribution network topology model, the grid is traversed from the fault point. The switches on both sides of the fault point are the fault loss area, the downstream of the fault area to the contact switch is the non-fault loss area, and the upstream of the fault point to the substation is the live area. The purpose of load transfer is to restore the power supply in the non-fault loss area.

\subsection{Path search for load transfer}

\subsubsection{Coding of real numbers based on loops}

In order to reduce the solution space, a real number coding method based on the distribution network loop is proposed. The actual wiring group is composed of multiple radial power supply lines interconnected. If a connecting branch is closed, a loop is formed between the two power supply lines. Therefore, a branch must be disconnected in this loop to restore the radial shape of each power supply line. The coding rule is: first, close the tie switch closest to the power-loss area to restore the wiring group to the normal operating topology. Then the remaining connecting switches of the wiring group are numbered with natural numbers, and each switch is individually numbered (from 1 to the total number of switches in the loop) in the loop determined by each connecting branch. A loop defined by a connecting branch corresponds to a locus on the chromosome, and the value of this bit is the switch number that is turned on in the loop network. The value of the gene position is a positive integer, and the range is from 1 to the total number of switches in the loop.

\subsubsection{Breadth first search (Radiation judgment)}

For the chromosomes obtained by the above coding method, there are still a large number of infeasible solutions, which need to be identified. Therefore, breadth first search is used to judge the radiation pattern of the generated topology. The radial operation constraint of distribution network can be equivalent to the following two conditions:

- $\quad$ Number of branches $=$ Number of nodes -1 .

- There is no electrical island node in the distribution network topology.

Only when randomly generated chromosomes meet the above two conditions can genetic manipulation be carried out. For chromosomes that do not meet the radiative check, they need to be rebuilt until they pass the check.

\subsubsection{Fast non-dominant hierarchical sorting}

Load transfer is a multi-objective optimization problem. In multi-objective programming, there are conflicts and incomparability between objectives. Pareto put forward the concept of multi-objective non-dominant solution in 1986: if two solutions S1 and S2 are superior to S2 for all objective functions, then S1 governs S2. If the solution of $\mathrm{S} 1$ is not dominated by other solutions, then $\mathrm{S} 1$ is called non-dominant solution, and the set of these non-dominant solutions is the so-called Pareto front.

At the same time, in order to ensure the diversity of solutions, it is hoped that the solutions in the same Pareto class can be separated from each other, and the concept of "crowding degree" is set up. The crowding distance of each individual is calculated by calculating the sum of the distance difference between the two adjacent individuals on each subobjective function.

$$
C D_{i}=\sum_{k}\left|f_{k}\left(x_{i+1}\right)-f_{k}\left(x_{i-1}\right)\right|
$$

Where, $f_{k}($.$) is the k$ th objective function.

The strategy of fast non-dominant hierarchical ordering mainly consists of the following two steps:

- According to Pareto's idea of optimal frontier, all individuals in the population are arranged hierarchically from small to large.

- There is no electrical island node in the distribution network topology.

\subsubsection{Elite selection}

Elite selection is an improvement over NSGA-II. The progeny is no longer generated by the parent directly by genetic operator, but by elite selection strategy. The parent population A firstly produces the offspring population $\mathrm{B}$ through selection, crossover and variation, and then gets the new parent population $(\mathrm{A}=\mathrm{A}$ Union $\mathrm{B})$ by taking union. At this point, the new population $\mathrm{A}$ includes both the parent generation and the offspring. On this basis, rapid non-dominant hierarchical sequencing was carried out for population $\mathrm{A}$, and excellent chromosomes were selected according to the grade and "crowding" size to participate in the next iteration as true descendants. Elite selection not only selects the excellent individuals produced in genetic operation, but also retains the original excellent individuals in offspring.

\subsubsection{Electrical power flow calculation}

Newton Raphson method is used to calculate the power flow, judge the constraint conditions and check the correctness of the transfer scheme.

\section{ANALYSIS OF EXAMPLES}

The test case selects a wiring group of a power supply unit in the distribution network of Taizhou City, Jiangsu 
Province. The CIM model is shown in the figure

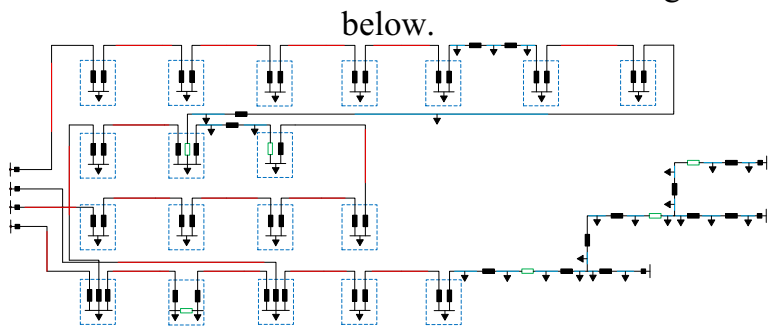

Figure 2. Node/Switch model of power supply unit wiring group in Taizhou distribution network.

Combine the node/switch model (CIM model) through the terminals and transform it into a bus/branch model, as shown in the figure below.

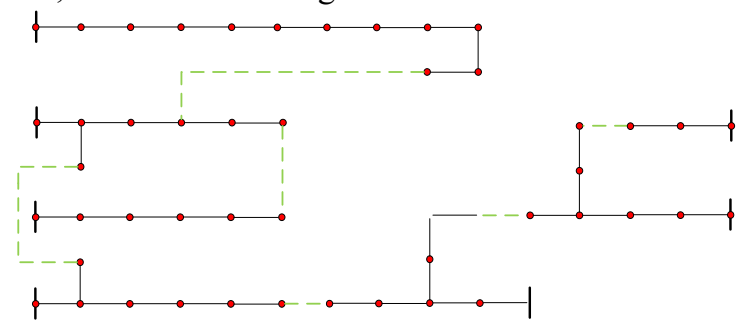

Figure 3. The bus/branch model of the power supply unit wiring group in Taizhou distribution network.

The model consists of 50 nodes, 43 closed branches and 7 connecting branches, and 7 power feeders supply power to the loads connected to it.

Set No. 31 branch fault, then the branch is disconnected and the fault is isolated. It is necessary to restore power to the non-faulty power-loss area downstream of the fault (area within the circle), as shown in the figure below.

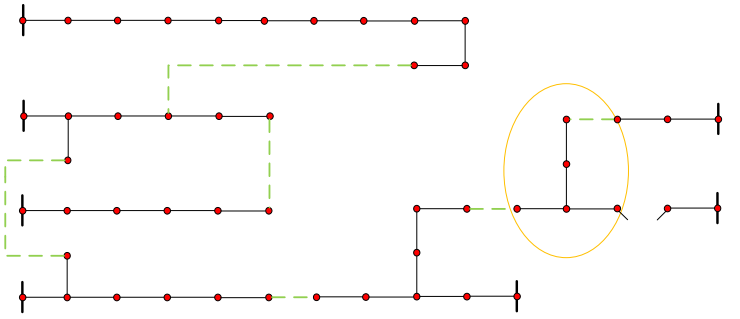

Figure 4. Test case wiring group faulty branch and non-faulty outage area.

The connecting branches directly connected to the fault area include branch 46 and branch 47 . Any branch can be closed to restore the radial shape of the network, and there are no electrical islands other than the disconnected branch.

Using the loop-based real number coding method, the number of loops is determined by the number of remaining unclosed contact switches as 5, that is, the gene position is 5 . Individually number the switches in the ring. The value range of each gene position of the chromosome is as follows:

Table 1. Range of chromosome locus.

\begin{tabular}{|c|c|c|}
\hline $\begin{array}{c}\text { Chromosomal } \\
\text { gene positions }\end{array}$ & $\begin{array}{c}\text { Minimum } \\
\text { chromosomal } \\
\text { gene positions }\end{array}$ & $\begin{array}{c}\text { Maximum } \\
\text { chromosomal } \\
\text { gene positions }\end{array}$ \\
\hline
\end{tabular}

\begin{tabular}{|c|c|c|}
\hline gene positions 1 & 1 & 25 \\
\hline gene positions 2 & 1 & 19 \\
\hline gene positions 3 & 1 & 9 \\
\hline gene positions 4 & 1 & 12 \\
\hline gene positions 5 & 1 & 8 \\
\hline
\end{tabular}

It can be seen from the table that after adopting the loop-based real number coding method, the size of the solution space of the load transfer problem is compressed to 410,400 . The solution space scale of the test cases of different coding methods is shown in Table 2 .

Table 2. Comparison of load transfer solution space scale of test cases with different coding methods.

\begin{tabular}{|c|c|c|}
\hline Encoding & Binary code & $\begin{array}{c}\text { Real number } \\
\text { coding based on } \\
\text { loop }\end{array}$ \\
\hline Number & $2^{49}$ & 410400 \\
\hline
\end{tabular}

It can be seen from the table that this type of coding method greatly reduces the size of the solution space, but there are still some infeasible solutions. When generating individuals, it is necessary to combine breadth first search for radial judgment and electrical islanding test. Through verification, the feasible solution and number statistics of the loop-based real number coding are as follows:

Table 3. Statistics on the number of feasible solutions of real number coding based on loop.

\begin{tabular}{|c|c|c|c|}
\hline $\begin{array}{c}\text { Distribution } \\
\text { of solutions } \\
\text { for test cases }\end{array}$ & $\begin{array}{c}\text { Solution } \\
\text { space }\end{array}$ & $\begin{array}{c}\text { Feasible } \\
\text { solution }\end{array}$ & Percentage \\
\hline Number & 410400 & 205308 & $50 \%$ \\
\hline
\end{tabular}

Set the population size to 200 , and the number of iterations is 20. Save the optimal Pareto frontier individuals that the algorithm runs for 5 times, and perform a quick non-dominated sorting on them again. That is, the optimal solution calculated by each algorithm is integrated, and an elite selection is performed again. This can avoid falling into a local optimum, and it is also conducive to reducing the scale of the optimal solution and optimizing the best solution. The calculated optimal results are as follows:

Table 4. NSGA-II best results.

\begin{tabular}{|c|c|c|}
\hline $\begin{array}{l}\text { Individual } \\
\text { number }\end{array}$ & Individual & $\begin{array}{c}\text { Corresponding to } \\
\text { open switch }\end{array}$ \\
\hline Individual 1 & $\begin{array}{lllll}25 & 16 & 9 & 12 & 8\end{array}$ & $\begin{array}{lllll}60 & 36 & 63 & 62 & 65\end{array}$ \\
\hline Individual 2 & $\begin{array}{lllll}15 & 7 & 8 & 12 & 8\end{array}$ & $\begin{array}{lllll}15 & 27 & 42 & 62 & 65\end{array}$ \\
\hline Individual 3 & $\begin{array}{lllll}14 & 19 & 4 & 12 & 8\end{array}$ & $\begin{array}{lllll}14 & 61 & 22 & 62 & 65\end{array}$ \\
\hline Individual 4 & 157978 & $\begin{array}{lllll}15 & 27 & 63 & 47 & 65\end{array}$ \\
\hline Individual 5 & 157378 & $\begin{array}{lllll}15 & 27 & 21 & 47 & 65\end{array}$ \\
\hline
\end{tabular}




\begin{tabular}{|c|l|llll|lllll|}
\hline Individual 6 & 16 & 7 & 8 & 8 & 8 & 16 & 27 & 42 & 48 & 65 \\
\hline Individual 7 & 16 & 8 & 4 & 7 & 8 & 16 & 28 & 22 & 47 & 65 \\
\hline Individual 8 & 16 & 8 & 3 & 7 & 8 & 16 & 28 & 21 & 47 & 65 \\
\hline
\end{tabular}

The fitness of the optimal individual is as follows:

Table 5. Optimal individual fitness.

\begin{tabular}{|c|c|c|c|}
\hline $\begin{array}{c}\text { Individual } \\
\text { number }\end{array}$ & $\begin{array}{c}\text { Voltage } \\
\text { deviation } \\
\text { rate }\end{array}$ & $\begin{array}{c}\text { Network } \\
\text { loss (p.u.) }\end{array}$ & $\begin{array}{c}\text { Switching } \\
\text { times }\end{array}$ \\
\hline Individual 1 & 0.00842391 & 0.00284843 & 3 \\
\hline Individual 2 & 0.00698578 & 0.00222135 & 7 \\
\hline Individual 3 & 0.0073707 & 0.00238258 & 5 \\
\hline Individual 4 & 0.0071031 & 0.00221312 & 7 \\
\hline Individual 5 & 0.00701303 & 0.00219939 & 9 \\
\hline Individual 6 & 0.00698631 & 0.00220494 & 9 \\
\hline Individual 7 & 0.00700088 & 0.00219991 & 9 \\
\hline Individual 8 & 0.00699187 & 0.00219991 & 9 \\
\hline
\end{tabular}

According to the recommendations of the combined business, the normalized voltage deviation rate, network loss, and switching times are respectively set as weights: $0.3,0.3,0.4$, and the optimal solution is calculated as individual 3. The corresponding transfer path is shown in Figure 5.

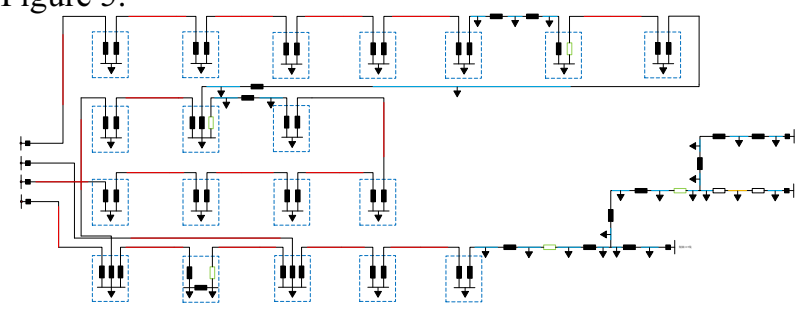

Figure 5. Node/switch model of test case wiring group after load transfer.

The bus/branch model corresponding to the CIM model of the test case after the load is transferred is shown in the figure below.

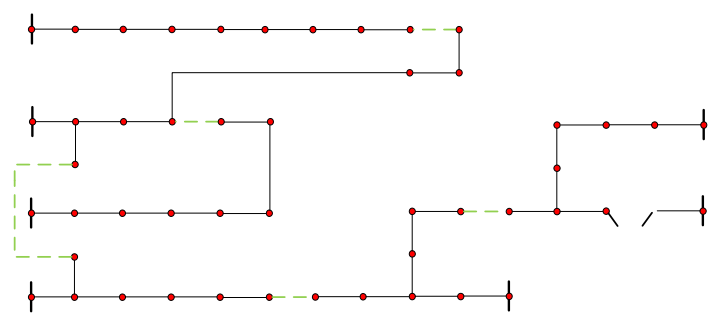

Figure 6. NSGA-II optimal forwarding path.

\section{Conclusion}

This paper uses the NSGA-II algorithm to analyze the load transfer of the medium voltage distribution network. By simulating faults, power supply can be restored to non-faulty power-loss areas. Establish an objective function with the smallest network loss, the smallest voltage deviation rate and the smallest number of switching actions. Use fast non-dominated sorting and elite selection strategies to iteratively search for the best. Finally, the Newton Raphson method is used to calculate the power flow and check the transfer plan. The results show that when NSGA-II is applied to the load transfer problem of the medium voltage distribution network, it can not only accurately compare the direct advantages and disadvantages of each objective function, but also comprehensively compare the advantages and disadvantages of the overall transfer plan. The algorithm ensures the diversity of the population individuals, makes the optimization process more evenly distributed in the solution space, and can quickly find the approximate global optimal transfer plan.

\section{References}

1. Ganiyu Adedayo Ajenikoko, Adebayo Wasiu Eboda, Tunde Samuel Adeyemi. A Genetic Algorithm Approach for Optimal Distribution System Network Reconfiguration. 2017, 7(9):1-10.

2. Dong-Li Duan, Xiao-Dong Ling, Xiao-Yue Wu, et al. Reconfiguration of distribution network for loss reduction and reliability improvement based on an enhanced genetic algorithm. 2015, 64:88-95.

3. Thuan Thanh Nguyen, Thang Trung Nguyen, Ngoc Au Nguyen, et al. Optimal Network Reconfiguration to Reduce Power Loss Using an Initial Searching Point for Continuous Genetic Algorithm. 2020, 2020

4. Damir Jakus, Rade Čađenović, Josip Vasilj, et al. Optimal Reconfiguration of Distribution Networks Using Hybrid Heuristic-Genetic Algorithm. 2020, 13(7)

5. N. Srinivas, Kalyanmoy Deb. Muiltiobjective Optimization Using Nondominated Sorting in Genetic Algorithms. 1994, 2(3):221-248. 